This is the final peer-reviewed accepted manuscript of:

Mentrelli, A. Shock structure in the 14 moment system of extended thermodynamics with high order closure based on the maximum entropy principle. Ricerche mat (2020).

The final published version is available online at: https://doi.org/10.1007/s11587020-00511-x

Rights / License:

The terms and conditions for the reuse of this version of the manuscript are specified in the publishing policy. For all terms of use and more information see the publisher's website. 


\title{
Shock Structure in the 14 Moment System of Extended Thermodynamics with High Order Closure Based on the Maximum Entropy Principle
}

\author{
Andrea Mentrelli \\ Department of Mathematics \& Alma Mater Research Center on Applied Mathematics $\mathrm{AM}^{2}$ \\ University of Bologna
}

\begin{abstract}
An analysis of the shock structure in the 14 moment system of Extended Thermodynamics with first, second and third order closure based on the maximum entropy principle (MEP) is presented, as a follow up of a recent investigation of the shock structure in the 13 moment system with first and second MEP-based closure.

It is seen that when adopting higher order closures, the strength of the subshock that appears in the shock structure profile for large enough Mach numbers is remarkably reduced with respect to what is found with the first order closure, and the overall profile of the shock structure solution is in better agreement with experimental results.
\end{abstract}

\section{Introduction}

Rational Extended Thermodynamics (RET) is a phenomenological theory originally developed for monatomic rarefied gases $[1,2]$, which has gained a renewed attention in recent years after being generalized to the case of polyatomic gases [3-6].

Originally intended as a generalization of the theory of Thermodynamic of Irreversible Processes (TIP), RET belongs to the realm of continuum theories, but its mathematical structure is closely connected to kinetic theory: The systems of first-order balance laws that distinguish the theory are in fact intimately related to the systems of velocity moments obtained starting from the Boltzmann equation.

One remarkable feature of RET, which derives from its relationship with kinetic theory, is that it is applicable to non-equilibrium states for which classical continuum theory fails, namely states with Knudsen numbers larger than those for which classical continuum theories are suitable. The original approach of RET to the problem of the closure is based on universal principles of continuum physics, i.e. the entropy principle, the principle of objectivity and the principle of thermodynamical stability. This approach, which has proven to be powerful and useful in several applications [2], also has its own shortcomings, since as the number of moments increases, it

Published on Ricerche di Matematica (Springer); https://doi.org/10.1007/s11587-020-00511-x (DOI: 10.1007/s11587-020-00511-x) 
becomes more and more difficult to effectively be used. In these cases, the strict relation to kinetic theory provides methods and procedures useful to takle the problem of the closure: the classical entropy principle can be replaced by the so-called Maximum Entropy Principle (MEP) [7-10]. The two approaches have proven to be equivalent [11], but the second one turns out to be easier to use when large number of moments are taken into account. Moreover, thanks to the peculiar way in which MEP is exploited in RET (the solution of the entropy maximization problem is sought only in the neighborhood of the local equilibrium) the combination of RET theory and the maximum entropy principle avoid the major issues affecting traditional theories with non-linear closure of the moment equations, i.e. the non convergence of the moments for any truncation order $[11,12]$, and the non-convexity of the domain of definition of the flux of the last moment equation [13].

These desirable features of the linear MEP-based closure has its own price: the validity of the theory is now limited to the neighborhood of the local equilibrium state, and the resulting system of equations loses the property of global hyperbolicity. In addition, since the systems of moment equations of RET are hyperbolic with a convex entropy density at least in some neighborhood of the equilibrium state, it was proven [14] that when the shock speed exceeds the maximum characteristic velocity in the unperturbed equilibrium state, a subshock appears in the otherwise continuous shock structure profile. The so-called subshocks - at least in the monatomic gas case - appear as undesirable artificial discontinuities that are not actually observed in experimental results. The case of a polyatomic gas turns out to be quite different from the case of monatomic gas, as was pointed out for example, in [15-17].

In a recent paper [18], the effect of a second order closure on the extension of the hyperbolicity region was investigated in the case of the 13 moment system of equations (the so-called Grad system), leading to the remarkable indication that, at least in the case under study, an increase in the order of the closure is accompanied by an increase (and a dramatic change of topology) of the hyperbolicity region. The results presented in [18] has suggested to further investigate the effects of second and even higher order MEP-based closure of the moment equations.

In a recent study an investigation along those lines was started. The features and the behaviors of the shock-structure solution were analyzed for the one-dimensional 13 moment system when first and second order MEP-based closures are exploited [19].

It was shown that a second order closure is beneficial not only, as proven in [18], in terms of the increase of the hyperbolicity region, but also from the point of view of the reduction of the subshock strength which appears in far-from-equilibrium processes. Moreover, the comparison of the results obtained with first and second order closures to those obtained by means of kinetic theory, revealed that the second order closure provides predictions that are in far better agreement with those obtained in the framework of the Boltzmann/BGK model.

In this paper, the analysis is extended to the well-known case of the 14 moment equations, and along with first and second order MEP-based closures, a third order closure is proposed and investigated.

The main motivation for extending the study to the 14 moment system is twofold: form one side, it is legitimate to assume that an increase in the number of moments (i.e. in the rank of tensorial system) can be accompanied by an increase in the order of the closure, and a third order closure is proposed for the first time. The second motivation relies in the fact that the 14 moment system can be regarded as more meaningful, from a physical point of view, than 
the 13 moment system. This consideration is based on a recent pivotal study by Pennisi and Ruggeri [20] who considered the classical limit of the moments associated with the relativistic Chernikov-Boltzmann equation and found an unexpected and intriguing way of building the hierarchy of moment equations, which leads to a natural and well identified sequence of moment systems of increasing order for the classical moments for both monatomic and polyatomic rarefied gases. This natural sequence of increasing-order moment equations does not include the 13 moment system of equations (Grad system), but includes the 14 moment system. The latter has been largely studied over the past decades, but the community was perhaps unaware of the meaningful role of the 14 moment system that is now recognized as a natural system emerging from the classical limit of the relativistic case.

The rest of the paper is organized as follows. In Section 2, the 14 moment system of a monatomic gas is recalled, and in Sections 2.1,2.2, and 2.3 the expressions of the closing fluxes that are obtained exploiting the maximum entropy principle with, respectively, first, second and third order closures are given for the one-dimensional case. The technique for finding the closure of the moment equations at various different orders will not be outlined here, since it has been extensively described elsewhere [19]. In Section 3, the basic elements of the shock structure solution of a first-order balance law system are recalled, and the systems of ordinary differential equations that allow to obtain such a solution in the case of the 14 moment system of a monatomic gas are provided for the first and second order closures (respectively, in Section $3.1,3.2$ ), being the equations for the third order quite long and not providing further insight (the structure is similar to those of the first two orders). In Section 4, the Rankine-Hugoniot compatibility conditions that permit to analyze the subshock formation in the case in which the shock structure solution is not continuous are briefly outlined. Details concerning the emerging systems of equations are not provided since they can be easily constructed following the procedure described in [19]. In Section 5, a selection of the numerical results obtained for the shock structure solution of the 14 moment system of a monatomic gas for various Mach numbers are shown. Finally, in Section 6, some concluding remarks on the present investigation are outlined.

\section{Moment System of a Monatomic Gas}

Following the general theory and adopting the same notation as in [19], the system of moment equations obtained taking the full tensorial equations of rank 0,1 and 2, augmented by the trace of the tensorial equation of rank 3 , and by the double trace of the tensorial equation of rank 4 is the well-known 14 moment system, first proposed by Kremer [21].

The 14 moment system is a first-order balance law system obtained with the generating weights $\boldsymbol{\Phi}=\left(1, c_{i}, c_{i} c_{j}, c_{k} c_{k} c_{i}, c_{k} c_{k} c_{l} c_{l}\right)^{T}$. In the one-dimensional case $(i, j=1)$ this system, reads:

$$
\partial_{t} \mathbf{u}+\partial_{x} \mathbf{F}=\mathbf{P}
$$


with

$$
\begin{gathered}
\mathbf{u}=\left(\begin{array}{c}
F \\
F_{1} \\
F_{k k} \\
F_{11} \\
F_{k k 1} \\
F_{k k l l}
\end{array}\right)=\left(\begin{array}{c}
\hat{F} \\
v \hat{F} \\
v^{2} \hat{F}+\hat{F}_{k k} \\
v^{2} \hat{F}+\hat{F}_{11} \\
v^{3} \hat{F}+2 v^{2} \hat{F}_{k k}+2 v \hat{F}_{11}+v \hat{F}_{k k}+\hat{F}_{11}+4 v \hat{F}_{k k 1}+\hat{F}_{k k l l 1}
\end{array}\right), \\
v \hat{F} \\
v^{2} \hat{F}+\hat{F}_{11} \\
\mathbf{F}=\left(\begin{array}{c}
F_{1} \\
F_{11} \\
F_{k k 1} \\
F_{111} \\
F_{k k 11} \\
F_{k k l l 1}
\end{array}\right)=\left(\begin{array}{c}
v^{3} \hat{F}+2 v \hat{F}_{11}+v \hat{F}_{k k}+\hat{F}_{k k 1} \\
v^{3} F+3 v F_{11}+F_{111} \\
v^{4} F+5 v^{2} F_{11}+v^{2} F_{k k}+2 v F_{111}+2 v F_{k k 1}+F_{k k 11} \\
v^{5} F+8 v^{3} F_{111}+2 v^{3} F_{k k}+6 v^{2} F_{k k 1}+4 v^{2} F_{111}+ \\
+4 v F_{k k 11}+v F_{k k l l}+F_{k k l l 1}
\end{array}\right),
\end{gathered}
$$

and

$$
\mathbf{P}=\left(\begin{array}{c}
0 \\
0 \\
0 \\
P_{11} \\
P_{k k 1} \\
P_{k k l l}
\end{array}\right)=\left(\begin{array}{c}
0 \\
0 \\
0 \\
\hat{P}_{11} \\
2 v \hat{P}_{11}+\hat{P}_{k k 1} \\
4 v^{2} \hat{P}_{11}+4 v \hat{P}_{k k 1}+\hat{P}_{k k l l}
\end{array}\right)
$$

Since, for a monatomic gas, $\hat{F}=\rho, \hat{F}_{11}=p-\sigma, F_{k k}=3 p, F_{k k 1}=2 q$, and writing $\hat{F}_{k k l l}=$ $15 p^{2} / \rho+\Delta$ (being $\Delta$ the non equilibrium part of the internal moment $\hat{F}_{k k l l}$ ), Eq. (2) and Eq. (3) can be written as

$$
\begin{gathered}
\mathbf{u}=\left(\begin{array}{c}
\rho \\
\rho v \\
\rho v^{2}+3 p \\
\rho v^{2}+p-\sigma \\
\rho v^{3}+5 p v-2 \sigma v+2 q \\
\mathbf{F}=\left(\begin{array}{c}
\rho v \\
\rho v^{4}+10 p v^{2}-4 \sigma v^{2}+8 q v+15 p^{2} / \rho+\Delta
\end{array}\right), \\
\rho v^{2}+p-\sigma \\
\rho v^{3}+5 p v-2 \sigma v+2 q \\
\rho p v-3 \sigma v+\hat{F}_{111} \\
\rho v^{4}+8 p v^{2}-5 \sigma v^{2}+4 q v+2 \hat{F}_{111} v+\hat{F}_{k k 11} \\
\rho v^{5}+14 p v^{3}-8 \sigma v^{3}+12 q v^{2}+15 p^{2} v / \rho+\Delta v+4 \hat{F}_{111} v^{2}+4 \hat{F}_{k k 11} v
\end{array}\right) .
\end{gathered}
$$

Adopting for the production terms the model [6]:

$$
\hat{P}_{11}=-\frac{\sigma}{\tau_{\sigma}}, \quad \hat{P}_{k k 1}=-\frac{2 q}{\tau_{q}}, \quad \hat{P}_{k k l l}=-\frac{\Delta}{\tau_{\Delta}},
$$


where $\tau_{\sigma}, \tau_{q}$, and $\tau_{\Delta}$ are relaxation times, Eq. (4) reads as follows:

$$
\mathbf{P}=\left(0, \quad 0, \quad 0, \quad \frac{\sigma}{\tau_{\sigma}}, \quad \frac{2 \sigma v}{\tau_{\sigma}}-\frac{2 q}{\tau_{q}}, \frac{4 \sigma v^{2}}{\tau_{\sigma}}+\frac{8 q v}{\tau_{q}}-\frac{\Delta}{\tau_{\Delta}}\right)^{T}
$$

and it becomes apparent that in order to close the system the internal moments $\hat{F}_{111}, \hat{F}_{k k 11}$, and $\hat{F}_{k k l l 1}$ must be written as functions of the densities.

In addition to the density $\rho$, momentum $\rho v$, and the pressure $p$, in the one-dimensional 14 moment system the non-equilibrium quantities $\sigma$ (shear stress), $q$ (heat flux) and $\Delta$ appear.

In contrast to the previous study [19] in which, in order to make a comparison with the kinetic model of the Boltzmann equation with the BGK approximation for the collision term (which involves one single relaxation time), the relaxation times appearing in the 13 moment system $\left(\tau_{\sigma}\right.$ and $\tau_{q}$ ) were assumed to be equal, in the following we shall let the three coefficients have different values, which is more adherent to physical reality.

\subsection{First Order Closure}

Making use of the theory illustrated in $[19,22]$, the maximum entropy principle with a first order closure, provides expressions for the moments $\hat{F}_{111}, \hat{F}_{k k 11}$, and $\hat{F}_{k k l l 1}$ (denoted, respectively, as $\hat{F}_{111}^{(1)}, \hat{F}_{k k 11}^{(1)}$ and $\hat{F}_{k k l l 1}^{(1)}$ ) which coincide with those obtained making use of the phenomenological closure developed in the context or RET [2], i.e.

$$
\hat{F}_{111}^{(1)}=\frac{6}{5} q, \quad \hat{F}_{k k 11}^{(1)}=5 \frac{p^{2}}{\rho}-7 \frac{p \sigma}{\rho}+\frac{\Delta}{3}, \quad \hat{F}_{k k l l 1}^{(1)}=\frac{28 p q}{\rho} .
$$

\subsection{Second Order Closure}

Adopting a second order closure, the algorithmic procedure provides the following expressions for the moments $\hat{F}_{111}, \hat{F}_{k k 11}$ and $\hat{F}_{k k l l 1}$, now denoted respectively as $\hat{F}_{111}^{(2)}, \hat{F}_{k k 11}^{(2)}$ and $\hat{F}_{k k l 11}^{(2)}$ :

$$
\begin{gathered}
\hat{F}_{111}^{(2)}=\hat{F}_{111}^{(1)}-\frac{36}{25} \frac{\sigma q}{p}, \quad \hat{F}_{k k 11}^{(2)}=\hat{F}_{k k 11}^{(1)}+\frac{\sigma^{2}}{\rho}+\frac{224}{75} \frac{q^{2}}{p}-\frac{14}{15} \frac{\sigma \Delta}{p}, \\
\hat{F}_{k k l l 1}^{(2)}=\hat{F}_{k k l l 1}^{(1)}-\frac{56}{5} \frac{\sigma q}{\rho}+\frac{28}{5} \frac{q \Delta}{p} .
\end{gathered}
$$

\subsection{Third Order Closure}

The third order closure obtained by means of the algorithmic procedure provides the following expressions for the moments $\hat{F}_{111}, \hat{F}_{k k 11}$ and $\hat{F}_{k k l l 1}$, now denoted respectively as $\hat{F}_{111}^{(3)}, \hat{F}_{k k 11}^{(3)}$ and 
$\hat{F}_{k k l l 1}^{(3)}$ :

$$
\begin{gathered}
\hat{F}_{111}^{(3)}=\hat{F}_{111}^{(2)}-\frac{24}{125} \frac{\rho \sigma q \Delta}{p^{3}}-\frac{99}{125} \frac{q \sigma^{2}}{p^{2}}+\frac{272}{625} \frac{\rho q^{3}}{p^{3}}, \\
\hat{F}_{k k 11}^{(3)}=\hat{F}_{k k 11}^{(2)}-\frac{28}{225} \frac{\rho \sigma \Delta^{2}}{p^{3}}+\frac{\sigma^{2} \Delta}{2 p^{2}}+\frac{56}{45} \frac{\rho q^{2} \Delta}{p^{3}}+\frac{14}{5} \frac{\sigma^{3}}{\rho p}+\frac{424}{125} \frac{\sigma q^{2}}{p^{2}}, \\
\hat{F}_{k k l l 1}^{(3)}=\hat{F}_{k k l l 1}^{(2)}+\frac{406}{225} \frac{\rho q \Delta^{2}}{p^{3}}-\frac{196}{25} \frac{\sigma q \Delta}{p^{2}}-\frac{204}{25} \frac{\sigma^{2} q}{\rho p}-\frac{168}{25} \frac{q^{3}}{p^{2}} .
\end{gathered}
$$

\section{Shock Structure Solution}

A shock structure solution of the one-dimensional system given in Eq. (1) is a continuous solution of the form

$$
\mathbf{u}=\mathbf{u}(\varphi), \quad \varphi=x-s t
$$

such that

$$
\lim _{\varphi \rightarrow-\infty} \mathbf{u}(\varphi)=\mathbf{u}_{1}, \quad \lim _{\varphi \rightarrow+\infty} \mathbf{u}(\varphi)=\mathbf{u}_{0}, \quad \lim _{\varphi \rightarrow \pm \infty} \frac{d \mathbf{u}}{d \varphi}=0,
$$

where $\mathbf{u}_{0}, \mathbf{u}_{1}$ are equilibrium states and $s$ is the speed of the travelling wave. Assuming, without loss of generality, that $s>0$, the states $\mathbf{u}_{0}$ and $\mathbf{u}_{1}$ are denoted, respectively, as unperturbed and perturbed states.

Writing the system (1) in the form

$$
\partial_{t} \mathbf{u}+\mathbf{A}(\mathbf{u}) \partial_{x} \mathbf{u}=\mathbf{P}(\mathbf{u}), \quad \mathbf{A}=\nabla_{\mathbf{u}} \mathbf{F}^{1},
$$

where $\mathbf{A}$ is the Jacobian of the flux $\mathbf{F}$ with respect to the field variables $\mathbf{u}$, it is seen [14] that a shock structure solution satisfies

$$
\frac{d}{d \varphi}\left(-s \mathbf{u}+\mathbf{F}^{1}\right)=\mathbf{P}(\mathbf{u}),
$$

or, equivalently,

$$
(-s \mathbf{I}+\mathbf{A}(\mathbf{u})) \frac{d \mathbf{u}}{d \varphi}=\mathbf{P}(\mathbf{u}) .
$$

The shock speed $s$ is often replaced by the so-called Mach number which, evaluated in the unperturbed state $\mathbf{u}_{0}$, is defined as $M_{0}=\left(s-v_{0}\right) / c_{0}$, where $v_{0}$ and $c_{0}$ are, respectively, the gas velocity and the sound velocity in the state $\mathbf{u}_{0}$ (in general, here and in the following the subscripts " 0 " and "1" denote a quantity evaluated, respectively, in the unperturbed and perturbed state).

The integration of the first three scalar equations of the system (8), representing the conservation laws of mass, momentum and energy, leads to the following relations between the two equilibrium states $\mathbf{u}_{0}$ and $\mathbf{u}_{1}$, i.e. the Rankine-Hugoniot compatibility conditions of the equilibrium subsystem [23]:

$$
\frac{\rho_{1}}{\rho_{0}}=\frac{4 M_{0}^{2}}{M_{0}^{2}+3}, \quad \frac{u_{1}}{u_{0}}=\frac{M_{0}^{2}+3}{4 M_{0}^{2}}, \quad \frac{p_{1}}{p_{0}}=\frac{5 M_{0}^{2}-1}{4},
$$

where, having introduced the relative velocity $u=v-s$, the quantities $\rho_{0}, u_{0}=v_{0}-s, p_{0}$ and $\rho_{1}, u_{1}=v_{1}-s, p_{1}$ are the density, relative velocity and pressure of, respectively, the unperturbed and perturbed states. 


\subsection{First Order Closure}

Introducing the following non-dimensional variables:

$$
\begin{gathered}
\hat{\varphi}=\frac{\varphi}{\tau_{\sigma} c_{0}}, \quad \hat{\rho}=\frac{\rho}{\rho_{0}}, \quad \hat{u}=\frac{u}{c_{0}}=-M_{0} \frac{u}{u_{0}}, \quad \hat{p}=\frac{p}{p_{0}}, \quad \hat{\sigma}=\frac{\sigma}{p_{0}}, \\
\hat{q}=\frac{q}{p_{0} c_{0}}, \quad \hat{\Delta}=\frac{\Delta}{c_{0}^{2}}, \quad r_{q}=\frac{\tau_{\sigma}}{\tau_{q}}, \quad r_{\Delta}=\frac{\tau_{\sigma}}{\tau_{\Delta}},
\end{gathered}
$$

the system of ordinary differential equations given in Eq. (8) with the flux F obtained by means of the first order closure, i.e. with the closing fluxes given by Eq. (5), is written in non-dimensional form after suitable manipulation as

$$
\begin{gathered}
\rho=-M_{0} / u \\
\sigma=-\frac{5}{3} M_{0}^{2}-\frac{5}{3} M_{0} u+p-1, \\
q=-\frac{5}{6} M_{0}^{3}-\frac{5}{3} M 0^{2} u-\frac{5}{6} M_{0} u^{2}-\frac{5}{2} M_{0}-\frac{3}{2} p u-u, \\
\frac{d}{d \varphi}\left(-9 M_{0}^{3}+27 M_{0}^{2} u+21 M_{0} u^{2}-27 M_{0}-81 p u / 5+81 u / 5\right)= \\
-15 M_{0}^{2}-15 M_{0} u+9 p-9, \\
\frac{d}{d \varphi}\left(\Delta M_{0}-16 M_{0}^{4} u-7 M_{0}^{3} u^{2}-21 M_{0}^{2} p u+4 M_{0}^{2} u^{3}-48 M_{0}^{2} u+\right. \\
\left.-204 M_{0} p u^{2} / 5-21 M_{0} u^{2} / 5+18 p^{2} u / 5-63 p u / 5\right)= \\
M_{0}\left(r_{q}\left(5 M_{0}^{3}+10 M_{0}^{2} u+5 M_{0} u^{2}+15 M_{0}+9 p u+6 u\right)+\right. \\
\left.-2 u\left(5 M_{0}^{2}+5 M_{0} u-3 p+3\right)\right), \\
\frac{d}{d \varphi}\left(u \left(35 \Delta M_{0}-210 M_{0}^{4} u+210 M_{0}^{3} p-220 M_{0}^{3} u^{2}-35 M_{0}^{2} u^{3}-\right.\right. \\
\left.\left.630 M_{0}^{2} u-498 M_{0} p u^{2}+630 M_{0} p-132 M_{0} u^{2}+315 p^{2} u\right)\right)= \\
-5 M_{0}\left(3 \Delta r_{\Delta}-4 r_{q} u\left(5 M_{0}^{3}+10 M_{0}^{2} u+5 M_{0} u^{2}+15 M_{0}+\right.\right. \\
\left.9 p u+6 u)+u^{2}\left(20 M_{0}^{2}+20 M_{0} u-12 p+12\right)\right)
\end{gathered}
$$

where the "hat" symbol on the quantities $\varphi, \rho, u, p, \sigma, q, \Delta$ was dropped for ease of notation and the unperturbed Mach number $M_{0}$ was used in place of the shock speed $s$.

\subsection{Second Order Closure}

When the flux $\mathbf{F}$ is obtained by means of the second order closure, i.e. making use of the closing fluxes given by Eq. (6), the system of ordinary differential equations in Eq. (8), written in non- 
dimensional form, becomes:

$$
\begin{aligned}
& \rho=-M_{0} / u \text {, } \\
& \sigma=-\frac{5}{3} M_{0}^{2}-\frac{5}{3} M_{0} u+p-1 \text {, } \\
& q=-\frac{5}{6} M_{0}^{3}-\frac{5}{3} M 0^{2} u-\frac{5}{6} M_{0} u^{2}-\frac{5}{2} M_{0}-\frac{3}{2} p u-u, \\
& \frac{d}{d \varphi}\left(\left(-450 M_{0}^{5}-1350 M_{0}^{4} u+45 M_{0}^{3} p-1350 M_{0}^{3} u^{2}-1620 M_{0}^{3}+405 M_{0}^{2} p u-\right.\right. \\
& -450 M_{0}^{2} u^{3}-2430 M_{0}^{2} u-15 M_{0} p u^{2}+135 M_{0} p-810 M_{0} u^{2}-810 M_{0}+ \\
& \left.\left.+81 p^{2} u+243 p u-324 u\right) /(25 p)\right)=-15 M_{0}^{2}-15 M_{0} u+9 p-9, \\
& \frac{d}{d \varphi}\left(\left(1050 \Delta M_{0}^{3}+1050 \Delta M_{0}^{2} u-405 \Delta M_{0} p+630 \Delta M_{0}+1400 M_{0}^{7}+\right.\right. \\
& +2900 M_{0}^{6} u+300 M_{0}^{5} u^{2}+8400 M_{0}^{5}+1935 M_{0}^{4} p u-2500 M_{0}^{4} u^{3}+ \\
& +10440 M_{0}^{4} u+4635 M_{0}^{3} p u^{2}-1300 M_{0}^{3} u^{4}+540 M_{0}^{3} u^{2}+12600 M_{0}^{3}+ \\
& -3375 M_{0}^{2} p^{2} u+1575 M_{0}^{2} p u^{3}+7830 M_{0}^{2} p u-1500 M_{0}^{2} u^{3}+5220 M_{0}^{2} u+ \\
& -378 M_{0} p^{2} u^{2}+2781 M_{0} p u^{2}+72 M_{0} u^{2}+405 p^{3} u-2025 p^{2} u+ \\
& -405 p u) /(225 p))=M_{0}\left(r _ { q } \left(5 M_{0}^{3}+10 M_{0}^{2} u+5 M_{0} u^{2}+15 M_{0}+9 p u+\right.\right. \\
& \left.+6 u)-2 u\left(5 M_{0}^{2}+5 M_{0} u-3 p+3\right)\right), \\
& \frac{d}{d \varphi}\left(\left(-3150 \Delta M_{0}^{4}-2100 \Delta M_{0}^{3} u+1050 \Delta M_{0}^{2} u^{2}-9450 \Delta M_{0}^{2}-6615 \Delta M_{0} p u-\right.\right. \\
& +1260 \Delta M_{0} u+5600 M_{0}^{7} u+17000 M_{0}^{6} u^{2}+6300 M_{0}^{5} p u+17400 M_{0}^{5} u^{3}+ \\
& +33600 M_{0}^{5} u+28350 M_{0}^{4} p u^{2}+6200 M_{0}^{4} u^{4}+61200 M_{0}^{4} u^{2}+5670 M_{0}^{3} p^{2} u+ \\
& +37080 M_{0}^{3} p u^{3}+22680 M_{0}^{3} p u+200 M_{0}^{3} u^{5}+31320 M_{0}^{3} u^{3}+50400 M_{0}^{3} u+ \\
& +9180 M_{0}^{2} p^{2} u^{2}+13905 M_{0}^{2} p u^{4}+70470 M_{0}^{2} p u^{2}+3720 M_{0}^{2} u^{4}+ \\
& +30600 M_{0}^{2} u^{2}+14526 M_{0} p^{2} u^{3}+17010 M_{0} p^{2} u+22248 M_{0} p u^{3}+ \\
& \left.\left.+11340 M_{0} p u+4176 M_{0} u^{3}+5751 p^{3} u^{2}+5508 p^{2} u^{2}+2916 p u^{2}\right) /(45 p)\right)= \\
& -5 M_{0}\left(3 \Delta r_{\Delta}-4 r_{q} u\left(5 M_{0}^{3}+10 M_{0}^{2} u+5 M_{0} u^{2}+15 M_{0}+9 p u+6 u\right)+\right. \\
& \left.+u^{2}\left(20 M_{0}^{2}+20 M_{0} u-12 p+12\right)\right) \text {, }
\end{aligned}
$$

where, as before, the "hat" symbol was dropped from the quantities $\varphi, \rho, u, p, \sigma, q$ and the the shock speed $s$ was replaced by the unperturbed Mach number $M_{0}$.

Adopting the third order closure characterized by the closing fluxes given by Eq. (7), the system of ordinary differential equations in Eq. (8) preserves the same structure as the systems obtained for the first and second order closures but, being longer and cumbersome, is not reported here for the sake of conciseness. 


\section{Shock Structure Solution with Subshock}

Denoting with $\lambda_{0}^{(\max )}$ the maximum characteristic speed (i.e. the largest eigenvalue of the matrix A) in the unperturbed state $\mathbf{u}_{0}$, it was proven that when $s>\lambda_{0}^{(\max )}$, no continuous shock structure solution exists [14]. In this case, the solution develops a discontinuity known as subshock.

The study of the subshock features is based on the Rankine-Hugoniot compatibility conditions that must hold across the subshock front:

$$
-s[[\mathbf{u}]]_{*}+\left[\left[\mathbf{F}^{1}\right]\right]_{*}=0,
$$

where $[[\psi]]_{*}=\psi_{*}-\psi_{0}$ represents the jump across the discontinuity (subshock) of the generic quantity $\psi\left(\psi_{*}\right.$ denotes the value of the quantity $\psi$ evaluated in the perturbed state behind the discontinuity, i.e. in the state $\mathbf{u}_{*}$ connected to the unperturbed state $\mathbf{u}_{0}$ through the subshock).

According to the general theory of hyperbolic systems, shocks are one-parameter families of solutions corresponding to the bifurcated branches of the trivial solution (null shock) obtained when the shock parameter $s$ approaches a characteristic velocity of the system. It is known that, when $M_{0} \gtrsim \frac{1}{5} \sqrt{\sqrt{826}+49} \simeq 1.76=M_{0}^{c r}$, the so-called fast shock appears.

The features of the solution of the system of non-linear equations given in Eq. (9) for the 14 moment system when a first, second, and third order closures are exploited, could be studied along the lines presented in [19] for the case of the 13 moment system, restricted to only the first two order of the closure (for the linear closure of the 13 moment system, see also [24]).

Since the main focus of the investigation presented here is the qualitative analysis of the shock structure profile for the 14 moment system, the study of the strength of the subshock developing above the critical Mach number will not be further pursued here.

\section{Numerical results}

In order to investigate the properties of the shock structure solution with and without subshock obtained for the 14 moment system with first, second and third order MEP-based closures, the system of differential equations presented in Section 2 has been numerically solved for different values of the shock parameter, i.e. the unperturbed Mach number $M_{0}$.

In this section, a selection of the numerical results is presented as to show the impact of the chosen order of the closure (either first, second, or third) on the obtained shock structure profiles. To this aim, in the following the density and velocity profiles across the shock structure obtained numerically solving the 14 moment system with first, second, and thid order closures for $M_{0}=1.7,1.9,2.1$ are reported.

In Fig. 1, the case of the shock structure obtained with $M_{0}=1.7$ is presented. In agreement with the expectations [11,23], in this case the shock structure profile is continuous, namely it does not present any discontinuity connected with a subshock formation, being the unperturbed Mach number $M_{0}$ less than the critical value $M_{0}^{c r} \simeq 1.76$. The results obtained by means of a first order closure present a continuous but some somewhat rapid transition from the unperturbed field to the perturbed one in the foremost part of the shock profile. Such a sharp (but continuous) transition is smoothed out when the second order closure is adopted, leading to a 

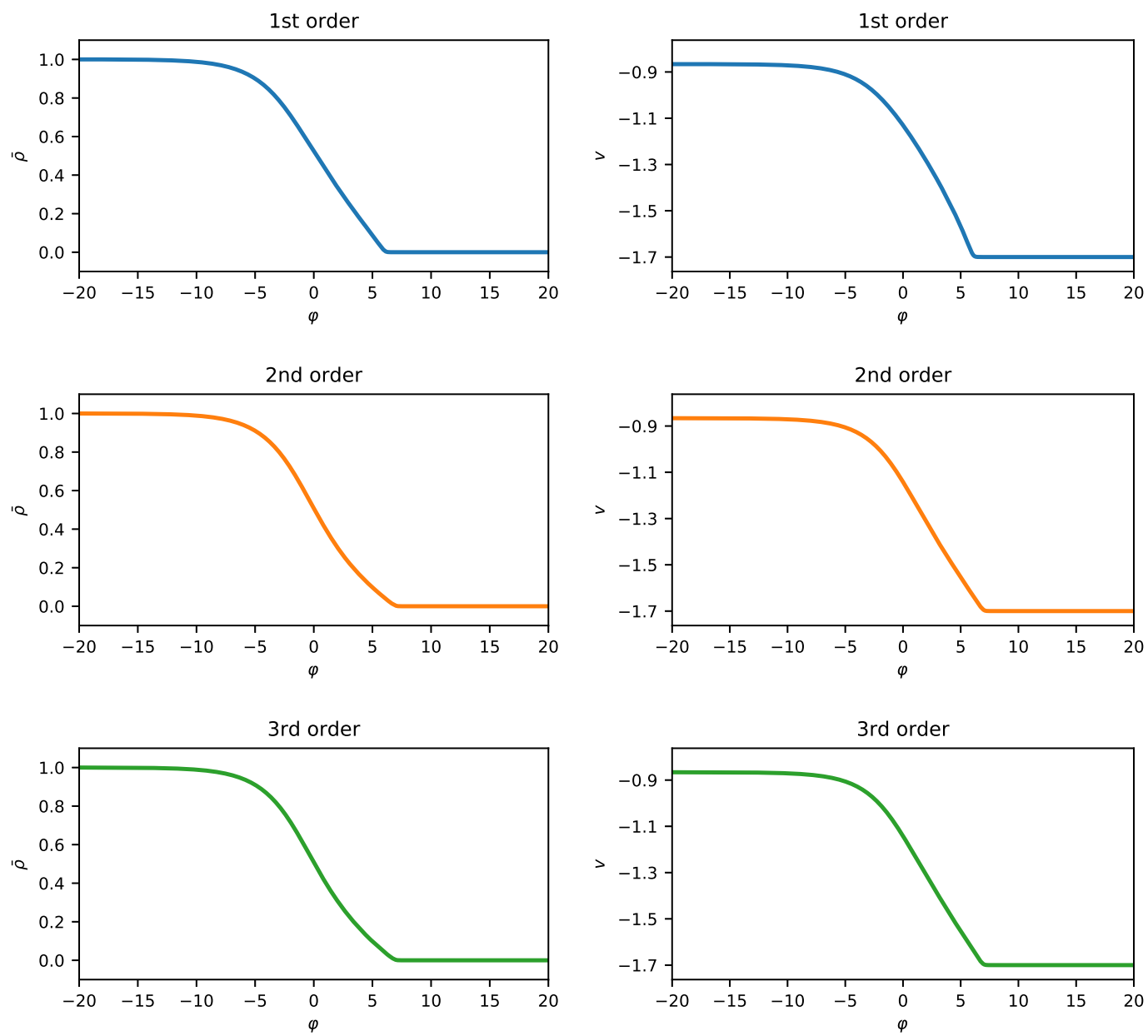

Figure 1: Rescaled mass density profile $\bar{\rho}=\left(\rho-\rho_{0}\right) /\left(\rho_{1}-\rho_{0}\right)$ (on the left) and relative velocity profile $u=v-s$ (on the right) of the shock structure solution obtained for $M_{0}=1.7$ in a monatomic gas with the 14 moment system with the first order (top row), second order (middle row) and third order (bottom row) MEP closure. 

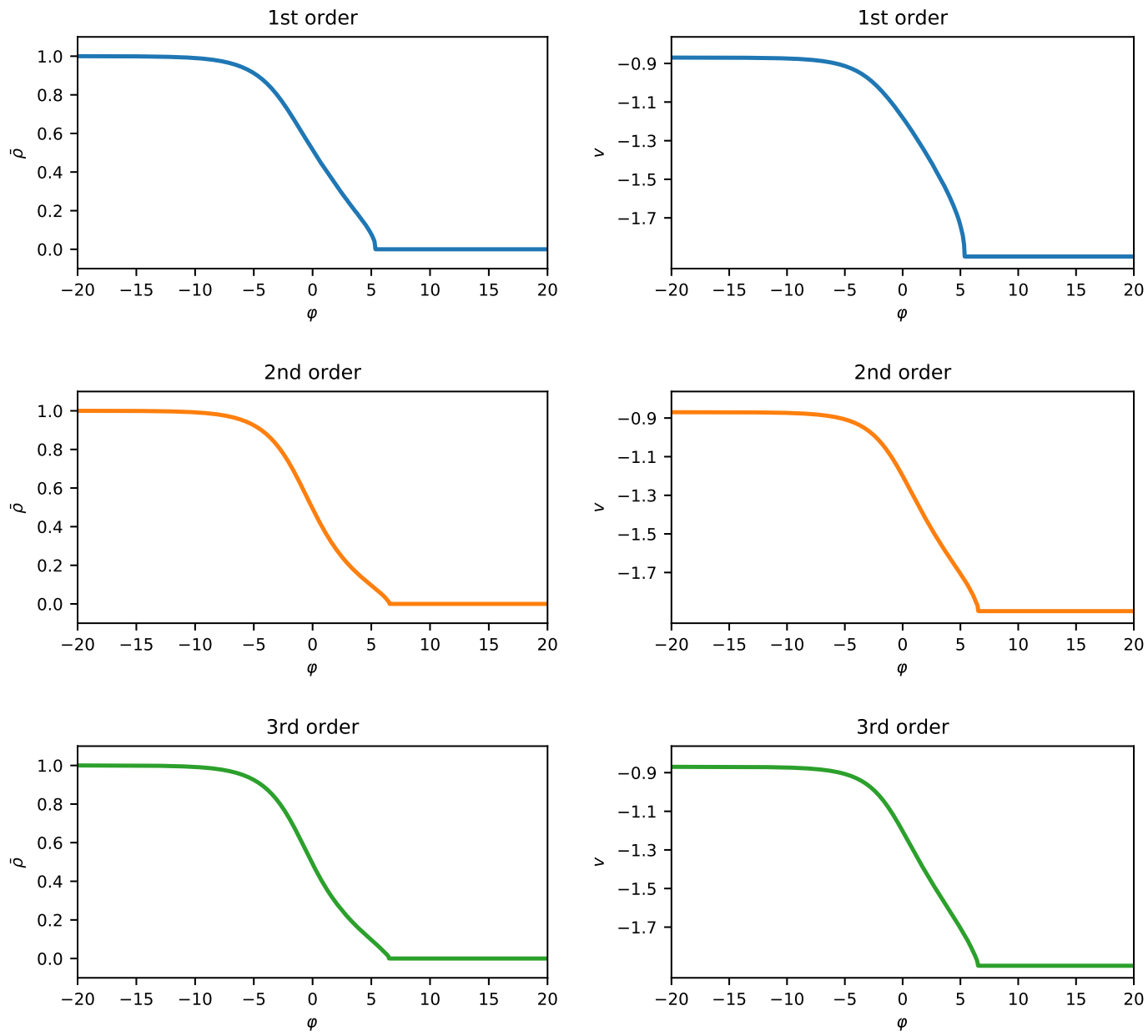

Figure 2: Rescaled mass density profile $\bar{\rho}=\left(\rho-\rho_{0}\right) /\left(\rho_{1}-\rho_{0}\right)$ (on the left) and relative velocity profile $u=v-s$ (on the right) of the shock structure solution obtained for $M_{0}=1.9$ in a monatomic gas with the 14 moment system with the first order (top row), second order (middle row) and third order (bottom row) MEP closure. 

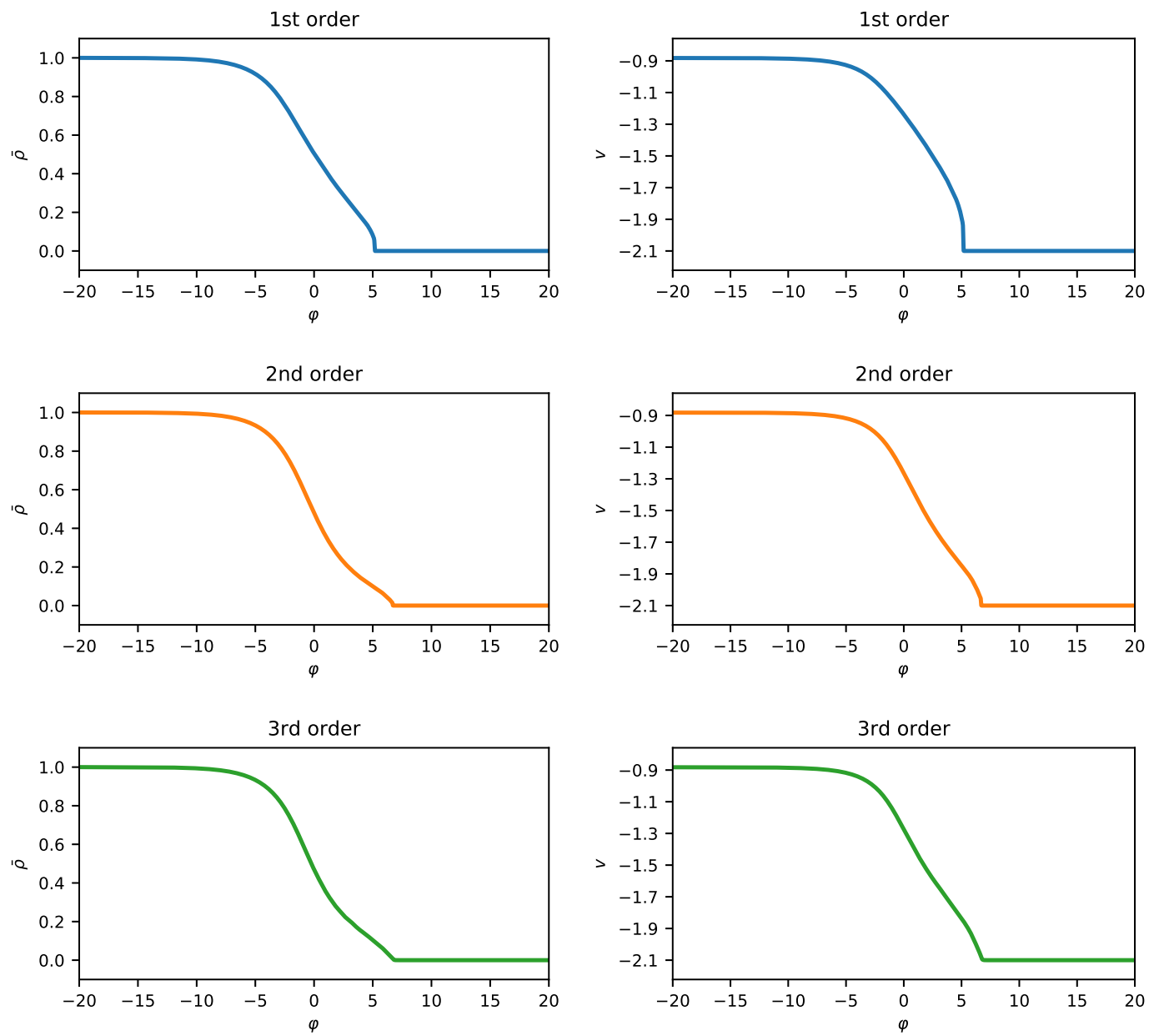

Figure 3: Rescaled mass density profile $\bar{\rho}=\left(\rho-\rho_{0}\right) /\left(\rho_{1}-\rho_{0}\right)$ (on the left) and relative velocity profile $u=v-s$ (on the right) of the shock structure solution obtained for $M_{0}=2.1$ in a monatomic gas with the 14 moment system with the first order (top row), second order (middle row) and third order (bottom row) MEP closure. 
shock profile which arguably matches better the expected real behavior of the monatomic gas. It is also observed that the difference in the shock structure profile between the second and the third order closure is barely noticeable.

In Fig. 2, the case of the shock structure obtained with $M_{0}=1.9$ is reported. In this case, the profile of the shock structure includes a discontinuity, i.e. a subshock, when the traditional first order closure of the 14 moment system is adopted. This is predicted by the theory, since in this case the unperturbed Mach number $M_{0}$ exceeds the critical value given by the largest characteristic velocity in the unperturbed state $\left(M_{0}^{c r} \simeq 1.76\right)$. The subshock formation is also evident in the shock structure profile obtained with the second and the third order closures, but in these cases the profiles are overall qualitatively different from the one obtained with the linear closure. In particular, it is seen that the strength of the subshock, i.e. the jump in the field variables across the subshock front is smaller than the one found making use of the linear closure. This is in agreement with the theoretical and numerical results obtained for the 13 moment system discussed in [19]. Even in this case, the shock structure profile obtained with the third order closure is similar (but distinguishable) from the one obtained with the second order closure.

Despite the fact that the overall shock structure profile always feature a subshock and therefore cannot qualitatively match the results that we would expect to find in a real physical situation, it is safe to say that the formation of the unphysical subshock discontinuity is a less prominent feature of the shock structure solution when the quadratic or cubic closures are adopted, in comparison to the solution obtained with the linear one.

It is worth noticing that the subshock formation, which takes place when the speed $s$ of the shock front exceeds the maximum characteristic velocity of the system evaluated in the unperturbed state [11] is independent from the chosen order of the closure. This is due to the fact that, as explained in [19], being the unperturbed state an equilibrium state, the fluxes of the moment equations are clearly independent from the chosen closure in any equilibrium state. As a consequence, the formation of a subshock is expected to be found independently from the order of the closure; the strength of the subshock, in contrast, strongly depends on the non-equilibrium fluxes, and hence on the adopted closure. For the 13 moment system, a detailed analysis of this behavior is presented in [19].

The case of a shock structure profile with $M_{0}=2.1$ is presented in Fig. 3 . As predicted by the theory, the shock profile obtained with the first order closure is affected by a rather pronounced unphysical jump in the foremost part of the shock profile.

When the second and third order closures are adopted, the solution presents a much reduced strength of the subshock, in comparison to the one obtained with the linear closure, and the second and third order closures start to differentiate one from the other. As a next step of the investigation, it would be interesting to compare the shock structure profiles obtained with large Mach numbers to the profile obtained in the framework of the kinetic theory, by means for example of a Boltzmann/ES-BGK model $[25,26]$.

\section{Conclusions}

The closure of the 14 moment system of a monatomic gas is generally obtained assuming a linear dependence of the closing fluxes on the non-equilibrium variables. In the framework of Rational 
Extended Thermodynamics (RET), this closure is obtained by means of the entropy principle, with a phenomenological approach, or equivalently by means of the maximum entropy principle (MEP).

The resulting 14 moment system has been largely investigated over the past decades, but only recently it was understood the full physical interest of the 14 moment system after realizing that this system, in contrast for example to the popular 13 moment system, is a natural system that can be obtained as the classical limit of the moments associated with the relativistic ChernikovBoltzmann equation [20].

An investigation on the qualitative features of the shock structure solution of the 14 moment system with second and third order closures based on the maximum entropy principle (MEP) is here presented, and the results are compared to those obtained with the traditional first order closure. It is remarkable that the subshock that appears in the shock structure profile for large enough Mach numbers is noticeably reduced when the second and third order closures are exploited. A similar behavior was already observed in the case of the 13 moment system, and suggests that the adoption of higher order closures along with increasing number of moments is potentially beneficial.

\section{Dedication}

It is my pleasure to dedicate this paper to Masaru Sugiyama and Giuseppe Toscani, who recently celebrated their 70th birthday. I have not been lucky enough to collaborate with Giuseppe Toscani yet, but having enjoyed several of his talks I have developed a true admiration for him. With Masaru Sensei Sugiyama I was more lucky, since I have had (and still have) the chance of collaborating with him in Nagoya and Bologna over the years. Not only have I learned a lot from him, but I also have the most sincere esteem of him. I sincerely wish to both of them all the best.

\section{Acknowledgements}

This work has been partially supported by GNFM/INdAM and by the Italian MIUR through the PRIN2017 project "Multiscale phenomena in Continuum Mechanics: singular limits, offequilibrium and transitions"(project number: 2017YBKNCE).

The author states that there is no conflict of interest.

\section{References}

[1] Müller, I., Ruggeri, T.: Extended Thermodynamics. Springer, New York (1993)

[2] Müller, I., Ruggeri, T.: Rational Extended Thermodynamics. 2nd Ed., Springer, New York (1998)

[3] Arima T., Taniguchi S., Ruggeri T., Sugiyama M.: Extended thermodynamics of dense gases. Continuum Mech. Thermodyn. 24, 271-292 (2012)

[4] Arima T., Taniguchi S., Ruggeri T., Sugiyama M.: Monatomic rarefied gas as a singular limit of polyatomic gas in extended thermodynamics. Phys. Lett. A 377, 2136-2140 (2013) 
[5] Arima T., Mentrelli, A., Ruggeri, T.: Molecular extended thermodynamics of rarefied polyatomic gases and wave velocities for increasing number of moments. Ann. Phys. 345, 111-140 (2014)

[6] Ruggeri, T., Sugiyama, M.: Rational Extended Thermodynamics Beyond the Monatomic Gas. Springer, Basel (2015)

[7] Jaynes, E. T.: Information theory and statistical mechanics. Phys. Rev. 106, 620-630 (1957)

[8] Jaynes, E. T.: Information theory and statistical mechanics II. Phys. Rev. 108, 171-190 (1957)

[9] Kogan, M. N.: Rarefied Gas Dynamics. Plenum Press, New York (1969)

[10] Kapur J. N.: Maximum Entropy Models in Science and Engineering. Wiley, New York (1989)

[11] Boillat, G., Ruggeri, T.: Moment equations in the kinetic theory of gases and wave velocities. Continuum Mech. Thermodyn. 9, 205-212 (1997)

[12] Levermore, C. D.: Moment closure hierarchies for kinetic theories. J. Stat. Phys. 83, 10211065 (1996)

[13] Junk, M.: Domain of Definition of Levermore's Five-Moment System. J. Stat. Phys. 93, 1143-1167 (1998)

[14] Boillat, G., Ruggeri, T.: On the Shock Structure Problem for Hyperbolic System of Balance Laws and Convex Entropy. Continuum Mech. Thermodyn. 5, 285-292 (1998)

[15] Taniguchi S., Arima T., Ruggeri T., Sugiyama M.: Thermodynamic theory of the shock wave structure in a rarefied polyatomic gas: Beyond the Bethe-Teller theory. Phys. Rev. E 89, 013025 (2014)

[16] Taniguchi S., Arima T., Ruggeri T., Sugiyama M.: Shock wave structure in rarefied polyatomic gases with large relaxation time for the dynamic pressure. J. Phys.: Conf. Ser. 1035, 012009 (2018)

[17] Ruggeri T., Taniguchi S.: Shock Waves in Hyperbolic Systems of Non-Equilibrium Thermodynamics. Applied Wave Mathematics II: Selected Topics in Solids, Fluids, and Mathematical Methods and Complexity. Mathematics of Planet Earth, Vol. 6(Eds. A. Berezovski, T. Soomere), Springer Verlag, 167-186 (2019)

[18] Brini, F., Ruggeri, T.: Second-order approximation of extended thermodynamics of a monatomic gas and hyperbolicity region. Continuum Mech. Thermodyn. 32, 23-39 (2020)

[19] Mentrelli, A., Ruggeri, T.: Shock Structure in Extended Thermodynamics with Second Order Maximum Entropy Principle Closure (submitted)

[20] Pennisi, S., Ruggeri, T.: Classical limit of relativistic moments associated with Boltzmann Chernikov equation: optimal choice of moments in classical theory (submitted) 
[21] Kremer, G. M., Extended thermodynamics of ideal gases with 14 fields, Ann. I. H. P., Phys. Theor. 45, 419-440 (1986)

[22] Brini, F., Ruggeri, T.: Entropy principle for the moment systems of degree $\alpha$ associated to the Boltzmann equation. Critical derivatives and non controllable boundary data. Continuum Mech. Thermodyn. 14, 165-189 (2002)

[23] Weiss W.: Continuous shock structure in extended thermodynamics. Phys. Rev. E 52, R5760-R5763 (1995)

[24] Ruggeri, T.: Shock Waves in Hyperbolic Dissipative Systems: Non Equilibrium Gases. Proceedings of the Euromech Colloquium 270 (Reggio Calabria, Italy; September 1990) (1991)

[25] Kosuge S., Aoki K.: Shock-wave structure for a polyatomic gas with large bulk viscosity. Phys. Rev. Fluids 3, 023401-1/42 (2018)

[26] Kosuge S., Kuo, H.-W., Aoki K.: A kinetic model for a polyatomic gas with temperaturedependent specific heats and its application to shock-wave structure. J. Stat. Phys. 177, 209251 (2019) 